\title{
Multi-disciplinary approach for enhancing orthodontic esthetics - case report
}

This article was published in the following Dove Press journal:

Clinical, Cosmetic and Investigational Dentistry

13 August 2015

Number of times this article has been viewed

\section{Ajit Kalia \\ Nasim Mirdehghan \\ Sonali Khandekar \\ Wasu Patil}

Department of Orthodontics, MA Rangoonwala Dental College, Pune, India

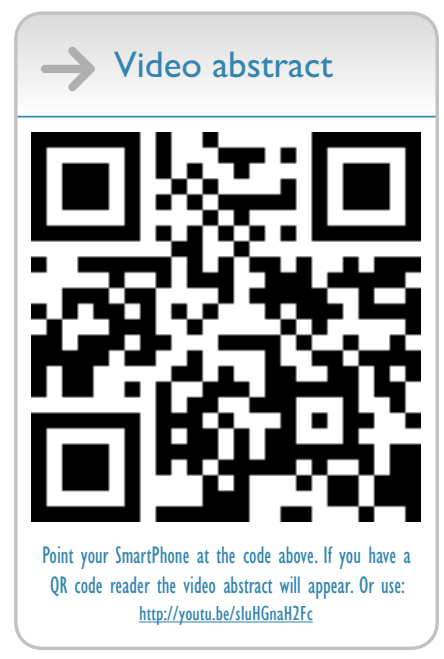

Correspondence: Ajit Kalia Department of Orthodontics, MA Rangoonwala Dental College, 2390-B, KB Hidayatullah Road, Azam Campus, Pune 4I I00I, India

Tel +9l 2026452288

Email msnasim@gmail.com
Background: In contemporary dental care, an increasing number of adult patients are now seeking orthodontic treatment with the primary motive of improvement in appearance and achievement of esthetic smile. Proper recognition of the dental and facial defects at the outset of treatment is the most important key to esthetic success and is essential in satisfying the patient's needs. By following the rules of Golden proportion smiles can be made attractive, harmonious, symmetrical, and proportionate.

Methodology: Consisted of 22-year-old girl who came for treatment of crowding in maxillary and mandibular arches, abnormal overjet and overbite and had unesthetic peg lateral.

Results: Non-extraction therapy was carried out since the patient had good soft tissue drape, alignment was achieved by expanding the arches and moving teeth in ideal axial inclination. Esthetic smile was attained using composite buildup of upper right peg lateral and minor adjunctive surgery (full thickness periodontal graft) on lower right central incisor.

Conclusion: The present case report orchestrates interrelationship between various branches of dentistry and orthodontics. It exhibits how multidisciplinary approach can be used to achieve ideal dental esthetics in a 22-year-old girl who was successfully treated for peg shaped lateral incisor, gingival recession, and unesthetic smile.

Keywords: adult orthodontics, peg lateral, gingival recession, golden proportion

\section{Introduction}

In the present era, orthodontics treatment spectrum has widened and is not limited to children and adolescents any more. The past two decades have seen a sudden surge in increasing number of adults seeking orthodontic interventions to enhance their esthetics.

In this appearance related society that we live in, young adults are becoming increasingly self-conscious about their smile. This trend toward a heightened awareness of esthetics has challenged the field of orthodontics to look into this aspect in a more organized and systematic manner. In order to achieve this, a good multi-disciplinary approach is essential.

Facial and dental appearance is broadly categorized into three parts; macro-esthetics, the examination of facial proportions in all three planes of space, mini-esthetics, the dentition in relation to the face, micro-esthetics, the teeth in relation to each other. This includes assessment of tooth proportions in height and width, gingival shape and contour, connectors and embrasures, black triangles, and tooth shade. In an ideal situation the apparent width of the lateral incisor is $62 \%$ of the width of the central incisor and the apparent width of the canine is $62 \%$ of the width of the lateral incisor. This ratio 
of recurring $62 \%$ is referred to as "Golden Proportion", 1.0:0.62:0.38:0.24. ${ }^{1}$

Frequently, the lateral incisors are disproportionately smaller than the central incisors. In such situations the golden proportion is an excellent guideline to determine what the post-treatment size of the lateral incisor should be. This size disparity can be corrected by reshaping or building the lateral incisors.

Gingival height, shape, and contour are an integral part of micro-esthetics and plays an important role in determining the post-treatment outcome.

Gingival recession is usually defined as a displacement of the gingival margin apically to the cementoenamel junction ${ }^{2}$ and is a mark of unesthetic smile. The prevalence of gingival recession is sex and population dependent and increases with age. ${ }^{3,4}$ The mandibular central incisors were the teeth with the highest prevalence of gingival recession. ${ }^{3,5}$ Usually, the lateral incisors maintain a more lingual position to the central incisors during eruption. A more pronounced labial position of the central incisors might result in a thin labial bone plate and apical migration of the marginal gingiva. The developmental position of the teeth also seems to be important as a predisposing factor to local gingival recession. ${ }^{5-7}$

Successful treatment outcome is an interdigitation of harmonious tooth proportion and ideal gingival morphology.

Multidisciplinary dental treatment is the new approach in the practice of dentistry as people now understand the requirements of a healthy and beautiful smile. More and more doctors are now doing specialty practice so as to deliver higher standards of quality dental care. Different specialists are now working as a cohesive unit under the same roof in order to deliver cost effective treatment plans so that they can pursue the objective of excellence in dentistry. This increases the horizons of treatment approach and is less stressful for the patients as well. The treatment approach is changed from paternalism to autonomy so as to meet the expectations of the patient.

A team of dental specialists can have a deeper insight into detailed diagnosis and resolve cumbersome dental issues effectively and fruitfully.

The present case report synchronizes interrelationship between various branches of dentistry and orthodontics to enhance esthetic outcomes.

\section{Diagnosis}

A 22-year-old girl came for orthodontic treatment with the chief complaint of crooked teeth and unesthetic smile. She had no significant medical or dental history. The patient had a straight profile with normal nose, competent lips, average mentolabial sulcus, and an average chin (Figure 1).

Intra-oral examination (Figure 2A-D) revealed Class I molar and canine relation on right and left side. The maxillary arch was broad with crowding in the anterior segment, retroclined incisors and peg shaped upper right lateral incisor. In the mandibular arch crowding was seen in the anterior segment with retroclined incisors and lingually placed lower right lateral incisor with a relatively thin cortical bone over lower right central incisor as observed clinically on inspection and palpation.

The occlusal features showed an overjet of $1 \mathrm{~mm}$, overbite of $5.5 \mathrm{~mm}$, and the lower midline was shifted to patient's right by $2 \mathrm{~mm}$. The curve of Spee was exaggerated.

Cephalometric analysis showed Class I skeletal base, an average growth pattern, and a good soft tissue drape (Table 1).

Model analysis revealed contracted upper and lower arches with space requirement of $3 \mathrm{~mm}$ in maxillary arch and $5.5 \mathrm{~mm}$ in mandibular arch. Bolton's analysis confirmed mandibular tooth material excess.

Other relevant radiographic findings included mesioangular lower right third molar and erupting upper and lower left third molars. Intra-oral periapical radiograph revealed thin cortical bone over lower right central incisor.

\section{Aims and objectives of treatment}

- To relieve crowding in maxillary and mandibular arches.

- Establishment of normal overjet and overbite.

- Esthetic consideration for peg lateral.

- Periodontal surgery for lower right central incisor if required.

- To establish long-term stability of results.

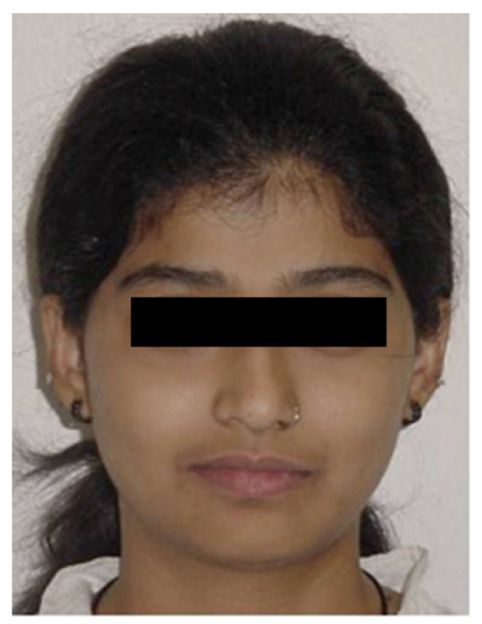

Figure I Pre-treatment frontol at rest. 

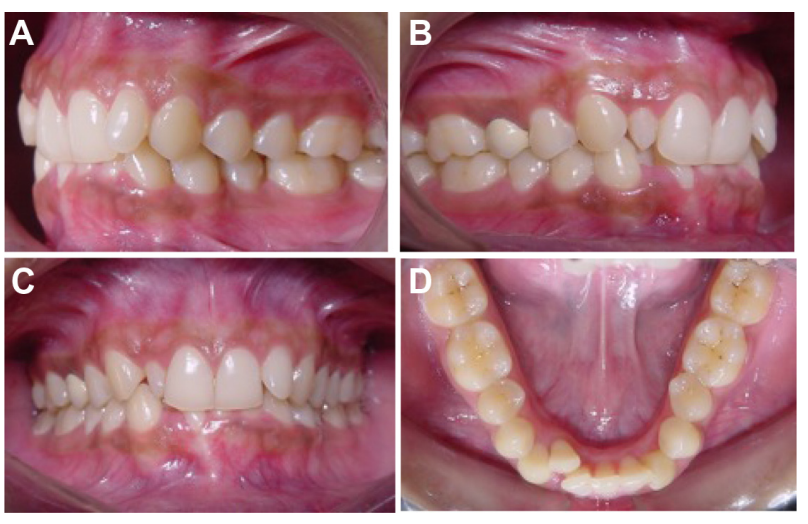

Figure 2 Pre-treatment intraoral photographs.

Notes: (A) Buccal left, (B) buccal right, (C) anterior view, (D) mandibular occlusal.

\section{Treatment plan}

Since the patient had good soft tissue drape, the treatment plan followed was non-extraction therapy. It was decided to gain space by proclination of the upper and lower incisors and by expanding the arches in order to achieve ideal axial inclination. Appliance used was pre-adjusted edgewise with slot size $0.022^{\prime \prime} \times 0.028$ ", Roth prescription (Mini-Roth series AO, American Orthodontics, Sheboygan, WI, USA).

Anchorage was reinforced by banding of upper and lower second molars. Ideal smile and esthetic needs of the patient were to be accomplished using composite buildup of upper right peg lateral.

Minor adjunctive surgery planned consisted of full thickness periodontal graft for lower right central incisor which had thin cortical plate. Proposed retention strategy was upper and lower bonded retainers and Hawley's wraparound for the upper arch.

Table I Values of pre-treatment cephalometric analysis

\begin{tabular}{lll}
\hline Variable & Pre-treatment & Normal \\
\hline Sagittal skeletal relationship & & \\
SNA & $81^{\circ}$ & $82^{\circ}$ \\
SNB & $79^{\circ}$ & $80^{\circ}$ \\
ANB & $2^{\circ}$ & $2^{\circ}$ \\
Wits appraisal & $+2.0 \mathrm{~mm}$ & $0 \mathrm{~mm}$ \\
Dental base relationship & & \\
Upper incisor to NA (mm/deg) & $+3 \mathrm{~mm} / 13^{\circ}$ & $4 \mathrm{~mm} / 22^{\circ}$ \\
Lower incisor to NB (mm/deg) & $+2 \mathrm{~mm} / 18^{\circ}$ & $4 \mathrm{~mm} / 25^{\circ}$ \\
Upper incisor to SN plane & $92^{\circ}$ & $102^{\circ}$ \\
Lower incisor to mandibular & $89^{\circ}$ & $90^{\circ}$ \\
plane angle (IMPA) & & \\
Dental relationship & & $131^{\circ}$ \\
Inter-incisal angle & $147^{\circ}$ & $0-2 \mathrm{~mm}$ \\
Lower incisor to APo line & $0 \mathrm{~mm}$ & $3.2 \pm 0.7 \mathrm{~mm}$ \\
Overbite & $+5.0 \mathrm{~mm}$ & $3.2 \pm 0.4 \mathrm{~mm}$ \\
Overjet & $+3.0 \mathrm{~mm}$ & \\
\hline
\end{tabular}

\section{Treatment progress}

Active treatment time was 21 months. Initially upper bands and brackets were placed, 0.016 heat activated nickel-titanium (HANT) (Ormco, Orange, CA, USA) wire was engaged. After 2 months of leveling and aligning, upper wire was changed to $0.018 \mathrm{SS}$ and then to $0.019 \times 0.025$ HANT.

Upper arch was progressed with $0.019 \times 0.025 \mathrm{SS}$ wire with reverse curve.

Lower bands and brackets were placed after 3 months and 0.016 HANT wire was engaged. Once alignment was accomplished, wire was changed to $0.018 \mathrm{SS}$ arch wire with active molar stops for expansion and proclination.

After expansion, consolidation of spaces in lower arch was done using elastomeric chain. Subsequently space was created for alignment of lingually placed lower right lateral incisor. A jockey arch wire -0.016 NITI wire was used to align the incisor into the arch. Final alignment of lower right lateral incisor was achieved by engaging lower 0.016 HANT wire with individual ligation.

After alignment of lower incisors $0.019 \times 0.025$ HANT wire was engaged which was moved on to lower $0.019 \times 0.025$ SS with reverse curve.

Torque expression was performed with upper and lower $0.021 \times 0.025 \mathrm{SS}$ wires which were kept for a span of 4 months. Final settling was done with 0.014 NITI wire and lower bonded retainers were fixed.

Buildup of upper right peg lateral incisor was done and subsequently upper bonded retainers were fixed (Figure 3A and B).

Patient was called for lower arch vestibuloplasty done under local anesthesia in order to reduce the strain on the attached gingiva (Figure 4). After 1 month of periodontal surgery healing - full thickness graft for lower right central incisor was done under local anesthesia (Figure 5A and B). The entire upper and lower arch brackets were debonded and wraparound Hawley's retainers were given. Postoperative recommendations in the form of oral hygiene regime and follow-up every 4 months (quarterly) for scaling were given to the patient in order to prevent failure of graft which
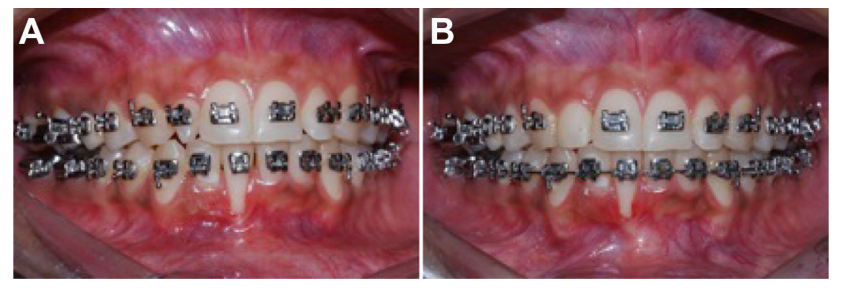

Figure 3 Before lateral incisor buildup (A) and after lateral incisor buildup (B). 


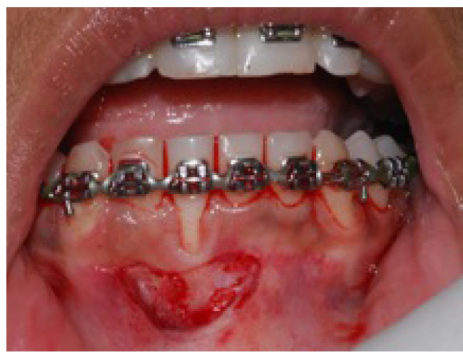

Figure 4 Lower arch vestibuloplasty.

was placed. The patient has been under regular follow-up for the last 18 months and there has been no sign of attachment loss.

\section{Treatment results}

Soft tissue drape and profile were maintained (Figure 6). Ideal smile esthetics was achieved by buildup of upper right lateral incisor and periodontal surgery of lower right central incisor. Crowding and deep bite were corrected and an ideal overjet and overbite were accomplished. Curve of Spee was leveled and midlines were coincided. Satisfactory functional and static occlusion was achieved (Figure 7A-D). Post-treatment OPG revealed adequate root parallelism (Figure 8) and lateral cephalogram showed adequate torque expression (Table 2).

\section{Discussion}

The occurrence of gingival recession has long been a subject of controversy. According to some studies proclination of the lower incisors during orthodontic treatment has been considered to be detrimental to periodontal health. ${ }^{8-10}$ In a recent review article, it was concluded that more proclined teeth had a higher occurrence or severity of gingival recession compared with less proclined or untreated teeth. However, the differences were small and the clinical consequences questionable. ${ }^{11}$ In contrast to this notion there are other studies which show that the risk of getting more severe gingival recession is increased in cases with retro-

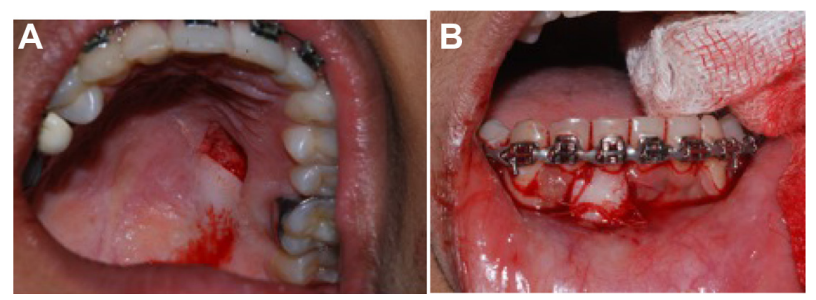

Figure 5 Free gingival graft taken from palate $(\mathbf{A})$ and full thickness graft on lower right incisor $\mathbf{B})$.

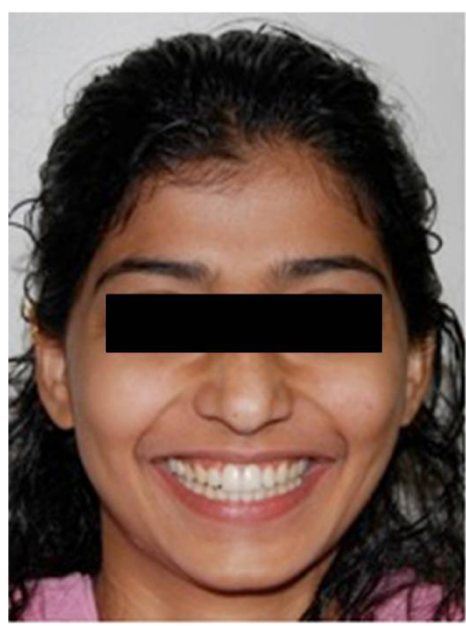

Figure 6 Post-treatment frontal smile photograph.

clined mandibular incisors and a mesial basal relationship of the jaws.

Various long-term studies indicate that in the presence of gingival recession, retroclination of the mandibular incisors in cases of mesial basal relation (Angle Class III) seems to increase the risk of a more severe gingival recession. Keeping the gingiva in the mandibular frontal area as healthy as possible during orthodontic treatment appears to be the determinant in the development of gingival recession. ${ }^{12}$ Inflammation seems to be a contributory factor for this condition. A careful examination of the buccal gingival and overlying alveolar bone in the lower incisors, before deciding how much the teeth need to be moved, seems important.

In the present case report the alveolar bone covering the lower right central incisor was thin both on inspection and palpation. Hence the option of full thickness graft was kept open from the initial phase of diagnosis and treatment planning.
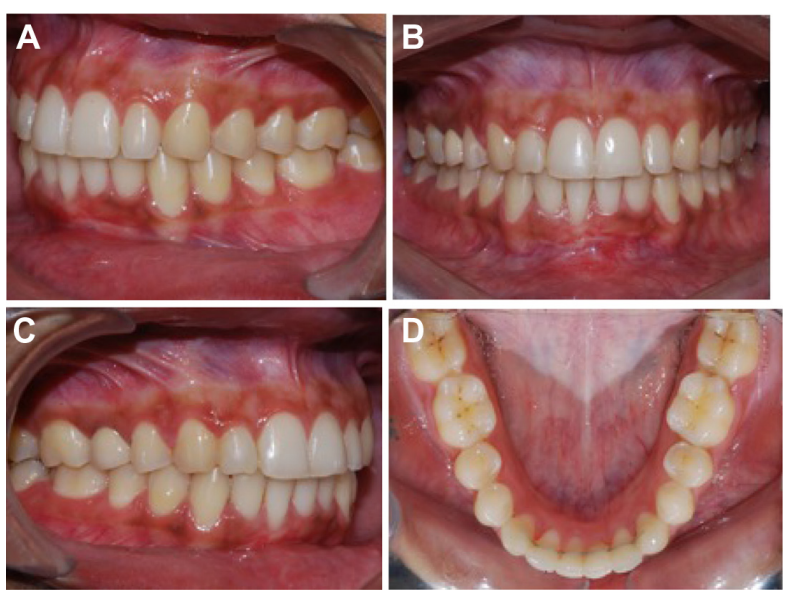

Figure 7 Post-treatment intraoral photographs.

Notes: (A) Buccal left, (B) anterior view, (C) buccal right and (D)mandibular occlusal. 


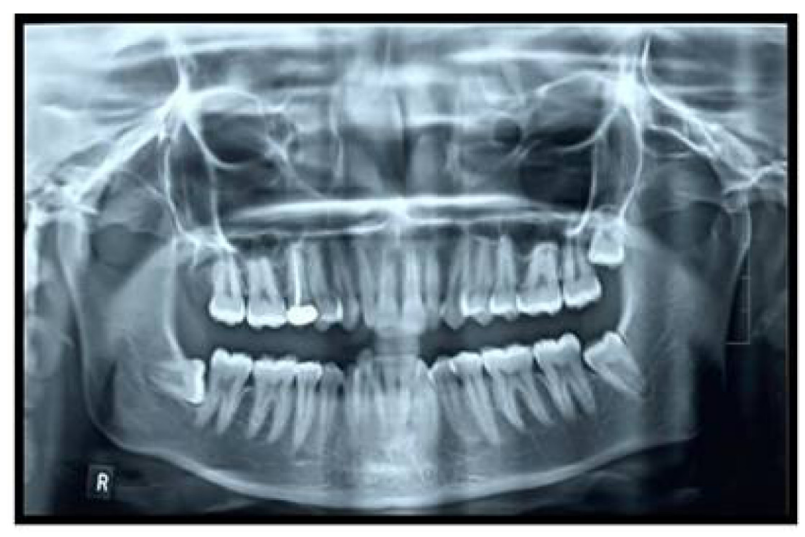

Pre-treatment OPG

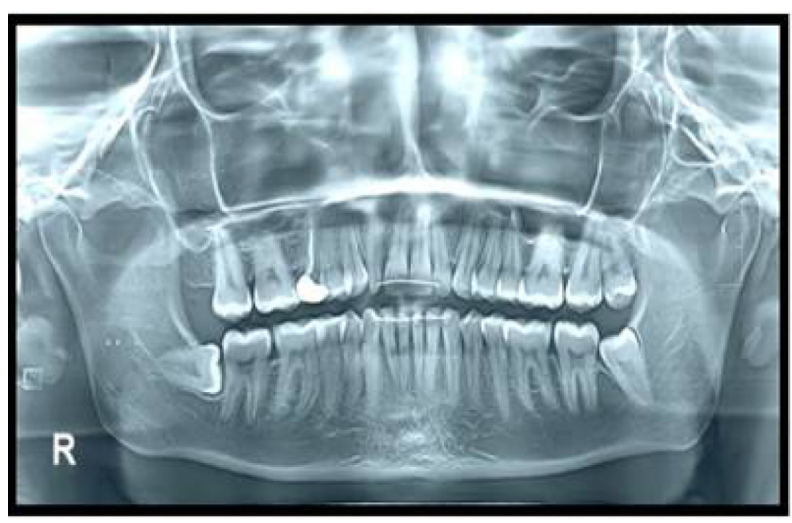

Post-treatment OPG

Figure 8 Pre-treatment OPG (top) and post-treatment OPG (bottom). Abbreviation: OPG, osteoprotegerin.

In the present case report the patient showed gingival recession with lower right central incisor at the end of orthodontic treatment which was corrected by periodontal surgery full thickness graft. Initially vestibuloplasty was done in order to increase the depth of lower arch vestibule and to reduce gingival strain on lower right central incisor. Gingival recessions are frequently associated with shallow vestibule or coronal frenum insertion especially in lower anterior region.

Poor mucogingival conditions may influence the passive surgical shift of the coronally advanced flap toward the cementoenamel junction and further decrease the vestibular depth.

Free gingival graft is the treatment of choice in cases demonstrating lack of keratinized tissue adjacent to the recession defect as it is effective in extending the fornix and in increasing both width and thickness of the keratinized tissue. ${ }^{13,14}$

However, it does not achieve predictable results in terms of complete root coverage with consequent impaired recovery from root sensitivity. ${ }^{14}$
Table 2 Values post-treatment cephalometric analysis

\begin{tabular}{|c|c|c|}
\hline Variable & Pre-treatment & Post-treatment \\
\hline \multicolumn{3}{|l|}{ Sagittal skeletal relationship } \\
\hline SNA & $81^{\circ}$ & $81^{\circ}$ \\
\hline SNB & $79^{\circ}$ & $79^{\circ}$ \\
\hline ANB & $2^{\circ}$ & $2^{\circ}$ \\
\hline Wits appraisal & $+2.0 \mathrm{~mm}$ & $+1.5 \mathrm{~mm}$ \\
\hline \multicolumn{3}{|l|}{ Dental base relationship } \\
\hline Upper incisor to NA (mm/deg) & $+3 \mathrm{~mm} / 13^{\circ}$ & $+7 \mathrm{~mm} / 28^{\circ}$ \\
\hline Lower incisor to NB (mm/deg) & $+2 \mathrm{~mm} / 18^{\circ}$ & $+7 \mathrm{~mm} / 30^{\circ}$ \\
\hline Upper incisor to SN plane & $92^{\circ}$ & $107^{\circ}$ \\
\hline $\begin{array}{l}\text { Lower incisor to mandibular } \\
\text { plane angle (IMPA) }\end{array}$ & $89^{\circ}$ & $102^{\circ}$ \\
\hline \multicolumn{3}{|l|}{ Dental relationship } \\
\hline Inter-incisal angle & $147^{\circ}$ & $118^{\circ}$ \\
\hline Lower incisor to APo line & $0 \mathrm{~mm}$ & $4 \mathrm{~mm}$ \\
\hline Overbite & $5 \mathrm{~mm}$ & $2 \mathrm{~mm}$ \\
\hline Overjet & $3 \mathrm{~mm}$ & $2 \mathrm{~mm}$ \\
\hline \multicolumn{3}{|l|}{ Vertical skeletal relationship } \\
\hline $\begin{array}{l}\text { Maxillary-mandibular planes } \\
\text { angle }\end{array}$ & $20^{\circ}$ & $21^{\circ}$ \\
\hline $\begin{array}{l}\text { SN plane-mandibular plane } \\
\text { angle }\end{array}$ & $27^{\circ}$ & $27^{\circ}$ \\
\hline Upper anterior face height & $56 \mathrm{~mm}$ & $60 \mathrm{~mm}$ \\
\hline Lower anterior face height & $64 \mathrm{~mm}$ & $71 \mathrm{~mm}$ \\
\hline Face height ratio & $56: 64$ & $60: 71$ \\
\hline Jarabak ratio & $82 / 118=69.5 \%$ & $90 / 130=69.2 \%$ \\
\hline Maxillary length & $86 \mathrm{~mm}$ & $95 \mathrm{~mm}$ \\
\hline $\begin{array}{l}\text { Mandibular length-effective } \\
\text { (McNamara) }\end{array}$ & $1 \mathrm{II} \mathrm{mm}$ & $121 \mathrm{~mm}$ \\
\hline \multicolumn{3}{|l|}{ Soft tissues } \\
\hline Lower lip to Ricketts E plane & $-1 \mathrm{~mm}$ & $-0.5 \mathrm{~mm}$ \\
\hline Naso labial angle & $97^{\circ}$ & $92^{\circ}$ \\
\hline
\end{tabular}

In addition, it is associated with poor esthetic appearance due to the unsatisfactory chromatic and texture tissue integration and the apical misalignment of the alveolar mucosa. ${ }^{15}$ Hence full thickness graft was chosen as it is devoid of above disadvantages.

Of the various adverse outcomes that may be encountered following periodontal surgery, the risk of infection stands at the forefront of concern to the surgeon, since infection can lead to poor healing outcomes. There are studies which confirm previous research demonstrating a low rate of postoperative infection following periodontal surgical procedures. Although the total postoperative complications were minimal in our case, literature reveals osseous surgery to be three times more likely than pure mucogingival surgery to cause complications of bleeding, infection, swelling or adverse tissue changes. Antibiotics are commonly used when performing certain regenerative and implant surgical procedures, studies suggest that there may be no benefit in using antibiotics for the sole purpose of preventing post-surgical infections. Further 
large-scale, controlled clinical studies are warranted to determine the role of perioperative antibiotics in the prevention of periodontal post-surgical infections. ${ }^{16}$ Studies suggest that the incidence and severity of complications following periodontal surgeries are minimal. ${ }^{17}$

Tooth proportion is one of the most important elements of anterior dental esthetics. Orthodontists often face the dilemma of correcting the disproportionate widths of anterior teeth. The rule of golden proportion becomes a savior in many critical situations. This ratio is an ideal treatment guide and can be mathematically defined as 1.0:0.62:0.38:0.24. ${ }^{1}$ When the rules of golden proportion are followed, the results obtained are naturally attractive and pleasing to the eye. It is imperative to restore the size of malformed lateral incisor after completion of orthodontic treatment for good overall treatment results which are harmonious, symmetrical, and proportionate.

Peg shaped lateral incisors occur in approximately $2 \%-5 \%$ of the general population, and women show a slightly higher frequency than men. Usually they are found equally on the right and left, uni- or bilaterally, however some studies have shown their bilateral occurrence slightly higher than the unilateral occurrence. When peg shaped laterals erupt in the mouth, esthetically it can be a disappointment to the patient that their teeth are not perfect or too small in comparison to the rest of the anterior teeth. Diagnosis is usually made clinically when peg lateral erupts. Treatment could be the combination of orthodontic treatment first to align the teeth in the arch, direct composite bonding onto peg laterals, indirect composite placement, bonded crowns, porcelain bonded to metal crowns, crown lengthening surgery to get better gingival heights then direct bonding, extractions, and implant placement.

Benefits of direct composite bonding include:

- preserved sound tooth structure; ${ }^{18}$

- can be placed directly onto the tooth surface;

- can place as a type of direct composite veneer;

- it is a conservative restoration;

- can easily change the emergence profile and angle;

- can alter the shape and length of the tooth;

- can close diastema;

- can be used as an interim restoration in an adolescent and added to as the gingival heights matures;

- can be repaired easily;

- can be polished and repolished to a high shine;

- long-lasting treatment option.

Disadvantages of direct composite bonding include:

- can chip and break;
- possible discoloration of older composite used;

- can develop marginal leakage, ${ }^{19}$

- can stain easily in those patients who smoke and have poor oral hygiene;

- can have a deleterious effect on gingival health in patients with poor oral hygiene. ${ }^{20}$

In the current case report lower arch was expanded to attain ideal inter canine width, overjet, and overbite. Non-extraction therapy was followed; full complement of dentition was maintained in order to bestow a fuller esthetic smile. Differential treatment option of lower right central incisor was discussed and rejected because of its evident disadvantage. Treatment with mandibular incisor extraction is not a popular technique in orthodontics because of its apparent shortcomings characterized by increase in overbite, overlap, unsatisfactory posterior occlusion, recurrence of mandibular crowding, and loss of esthetics of the inter dental papilla.

The present case report demonstrates the interrelationship between various specialties of dentistry to achieve harmonious results in the form of ideal symmetry, proportion, and esthetics.

\section{Conclusion}

Synchronizing various specialties with orthodontics in an organized and systematic manner is required to diagnose and resolve esthetic problems predictably. Our ultimate goal as orthodontist is to achieve pleasing composition in the smile by creating an arrangement of various esthetic elements. This case report suggests that the incidence and severity of complications following periodontal surgeries are minimal. The patient was happy with the overall treatment results and was satisfied with the pleasant and esthetic smile achieved at the end of the treatment. This article reviews the various principles which are important when creating an esthetic smile with satisfactory functional and static occlusion.

\section{Disclosure}

The authors have no conflicts of interest to disclose in this work.

\section{References}

1. Proffit W, Fields HW, Sarver DM. Orthodontic Diagnosis. In: Proffit W, Fields HW, Sarver DM, authors. Contemporary orthodontics Fourth edition. Elsevier Health Sciences; 2006:189.

2. Wennstrom JL. Mucogingival considerations in orthodontic treatment. Semin Orthod. 1996;2(1):46-54.

3. Albandar JM, Kingman A. Gingival recession, gingival bleeding and dental calculus in adults 30 years of age and older in the United States, 1988-1994. J Periodontol. 1999;70(1):30-43. 
4. Gorman WJ. The prevalence and etiology of gingival recession. J Periodontol. 1967;38(4):316-322.

5. Parfitt GJ, Mjör IA. A clinical evaluation of localized gingival recession in children. J Dent for Child. 1964;31:257-262.

6. Geiger AM. Mucogingival problems and the movement of mandibular incisors: a clinical review. Am J Orthod. 1980;78(5):511-527.

7. Wennström JL. Lack of association between width of attached gingiva and development of gingival recessions. A 5-year longitudinal study. J Clin Periodontol. 1987;14(3):181-184.

8. Årtun J, Krogstad O. Periodontal status of mandibular incisors following excessive proclination: a study in adults with surgically treated mandibular prognathism. Am J Orthod Dentofacial Orthop. 1987;91(13):225-232.

9. Hollender L, Ronnerman A, Thilander B. Root resorption, marginal bone support and clinical crown length in orthodontically treated patients. Eur J Orthod. 1980;2(4):197-205.

10. Fuhrmann R. Three-dimensional interpretation of labiolingual bone width of the lower incisors. J Orofac Orthop. 1996;57(3):168-185.

11. Joss-Vassalli I, Grebenstein C, Topouzelis N, Sculean A, Katsaros C. Orthodontic therapy and gingival recession: a systematic review. Orthod Craniofac Res. 2010;13(3):127-141.

12. Sperry TP, Speidel TM, Isaacson RJ, Worms FW. The role of dental compensations in the orthodontic treatment of mandibular prognathism. Angle Orthod. 1997;47(4):293-299.
13. Ward VJ. A clinical assessment of the use of the free gingival graft for correcting localized recession associated with frenal pull. J Periodontol. 1974;45(2):78-83.

14. Agudio G, Nieri M, Rotundo R, Cortellini P, Pini Prato G. Free gingival grafts to increase keratinized tissue: a retrospective long-term evaluation (10 to 25 years) of outcomes. J Periodontol. 2008;79(4): 587-594.

15. Kerner S, Sarfati A, Katsahian S, et al. Qualitative cosmetic evaluation after root-coverage procedures. J Periodontol. 2009;80(1):41-47.

16. Powell CA, Mealey BL, Deas DE, McDonnell HT, Moritz AJ. Postsurgical infections: prevalence associated with various periodontal surgical procedures. J Periodontol. 2005;76(3):329-333.

17. Curtis JW Jr, McLain JB, Hutchinson RA. The incidence and severity of complications and pain following periodontal surgery J Periodontol. 1985;56(10):597-601.

18. Izgi AD, and Emrah A. Direct restorative treatment of peg shaped maxillary lateral incisors with resin composite: A clinical report. Journal of Prosthetic Dentistry.1999;93:6 525-529.

19. Walls AW, Murray JJ, McCabe JF. Composite laminate veneers: a clinical study. J Oral Rehabil. 1988;15:439-454.

20. Greenwall L. Treatment options for peg-shaped laterals using direct composite bonding. International Dentistry SA. 2012;1:26-33.
Clinical, Cosmetic and Investigational Dentistry

\section{Publish your work in this journal}

Clinical, Cosmetic and Investigational Dentistry is an international, peer-reviewed, open access, online journal focusing on the latest clinical and experimental research in dentistry with specific emphasis on cosmetic interventions. Innovative developments in dental materials, techniques and devices that improve outcomes and patient satisfac-

\section{Dovepress}

tion and preference will be highlighted. The manuscript management system is completely online and includes a very quick and fair peerreview system, which is all easy to use. Visit http://www.dovepress. com/testimonials.php to read real quotes from published authors. 